\title{
Mechanical Thrombectomy in Basilar Artery Thrombosis: Technical Advances and Safety in a 10-Year Experience
}

\author{
Gunther Fesl - Markus Holtmannspoetter - Maximilian Patzig - Thomas E. Mayer • \\ Thomas Pfefferkorn · Hartmut Brueckmann • Gernot Schulte-Altedorneburg
}

Received: 28 July 2013/ Accepted: 17 November 2013/Published online: 23 January 2014

(C) Springer Science+Business Media New York and the Cardiovascular and Interventional Radiological Society of Europe (CIRSE) 2014

\begin{abstract}
Purpose Multiple endovascular devices have been used for mechanical thrombectomy (MT) in basilar artery occlusion (BAO) for $>10$ years. Based on a single-center experience during the course of one decade, we present data on safety and efficacy of previous MT devices compared with modern stent retriever and suction thrombectomy.

Materials and Methods Eighty-one patients (29 women, 52 men, mean age 61.5 years, range 17-90) with angiographically confirmed BAO that had been treated by MT between 2001 and 2011 were retrospectively evaluated. Patients in group $1(n=60)$ had been treated between 2001 and 2008 with different devices available at that time. Patients in group $2(n=21)$ had been treated by modern stent retriever or local suction devices between 2008 and 2011. Recanalization rate, needle to recanalization time, procedure-related complications, and distal embolization of thrombotic material were recorded and compared.
\end{abstract}

Gunther Fesl and Markus Holtmannspoetter contributed equally to this work.

G. Fesl $(\bowtie) \cdot$ M. Patzig $\cdot$ H. Brueckmann

Department of Neuroradiology, Klinikum Grosshadern, University of Munich, Marchioninistr. 15, 81377 Munich, Germany

e-mail: gunther.fesl@med.uni-muenchen.de

M. Patzig

e-mail: maximilian.patzig@med.uni-muenchen.de

H. Brueckmann

e-mail: hartmut.brueckmann@med.uni-muenchen.de

\section{Holtmannspoetter}

Department of Diagnostic Radiology, Rigshospitalet Copenhagen, Blegdamsvej 9, 2100 Copenhagen, Denmark

e-mail: markus.holtmannspoetter@ rh.regionh.dk
Results Recanalization rates of $95 \%$ were high in both groups. Procedure-related dissection $(n=5)$ and subarachnoid hemorrhage $(n=9)$ occurred in group 1 but not in group $2(p<0.016)$. Needle-to-recanalization time was less than half in group 2 compared with group 1 (54.6 vs. $132.3 \mathrm{~min}, p<0.01)$. Frequency of distal embolization was comparable in both groups $(47 \%)$.

Conclusion High recanalization rates have been achieved since the introduction of MT in BAO. However, modern stent retriever and suction devices allow for safer and more rapid recanalization compared with previous MT devices.

Keywords Mechanical thrombectomy - Basilar artery thrombosis - Safety - Long term experience

\section{Introduction}

Although the standard therapy, local intra-arterial fibrinolysis, has decreased mortality rates in acute true basilar artery occlusion (BAO) from 80 to $90 \%$ to approximately $60 \%$, acute basilar artery embolism is still a devastating

\footnotetext{
T. E. Mayer

Department of Neuroradiology, University of Jena, Erlanger

Allee 101, 07747 Jena, Germany

e-mail: t.e.mayer@med.uni-jena.de

T. Pfefferkorn

Department of Neurology, Klinikum Ingolstadt, Krumenauerstr.

25, 85049 Ingolstadt, Germany

e-mail: thomas.pfefferkorn@klinikum-ingolstadt.de

G. Schulte-Altedorneburg

Institute for Diagnostic and Interventional Radiology,

Neuroradiology and Nuclear Medicine, Klinikum München-

Harlaching, Sanatoriumsplatz 2, 81545 Munich, Germany

e-mail: gernot.schulte-altedorneburg@klinikum-muenchen.de
} 
life-threatening disease [1-4]. Furthermore, intra-arterial fibrinolysis has some limitations (e.g., low efficiency in cases of large thrombus load, frequent distal embolization, extensive time to recanalization) and potential intrinsic complications (i.e., intracranial bleeding) $[3,5,6]$.

During the last decade, several transarterial techniques for mechanical thrombectomy (MT), including suction, have been introduced in acute stroke therapy [7-11]. MT seems to be a more effective and probably less hazardous endovascular technique than intra-arterial fibrinolysis to achieve rapid and complete restoration of the blood flow, which is crucial for a favourable prognosis in patients with BAO [2-4, 6]. The recent MERCI [9, 10] and Penumbra [8] studies presented promising recanalization rates in the anterior and posterior circulation. Similar results have been reported in small studies and case reports considering the vertebrobasilar circulation alone [7, 11]. In contrast, procedure-related complications, such as dissection or perforation of the intracranial artery due to the stiffness of the thrombectomy device or the microwire, limit the benefit for patients' outcome and long-term prognosis [12].

The newest generation of MT devices are called "stent retrievers" [13-15]. Stent retrievers are self-expanding, fully retrievable, flexible, intracranial microstents [e.g., Solitaire (ev3 Endovasc. Inc, Plymouth, MN, USA), preset (Phenox GmbH, Bochum, Germany), Trevo pro (Concentric Medical Inc, Mountain View, CA, USA)] that allow for entrapment of the clot and subsequent removal of the embolic material under continuous aspiration. The Solitaire stent retriever is also suitable for permanent stent placement if needed [13].

The aim of the study was to analyze the efficacy and safety of different MT techniques in BAO. We present the interventional and periprocedural data of 81 patients treated by MT in a single stroke center during the last decade.

\section{Materials and Methods}

\section{Patients}

Eighty-one consecutive patients [ 29 women, 52 men, mean age 61.5 years (range 17-90)] with angiographically proven $\mathrm{BAO}$ treated with MT at one academic neurointerventional stroke center between 2001 and 2011 were retrospectively evaluated. According to the MT devices used, the patients were subdivided into two groups: Group 1 comprised 60 patients. They were treated by (1) AngioJet (Possis, Minneapolis, MN, USA; $n=27$ ), (2) percutaneous transluminal angioplasty (PTA) with $(n=8)$ or without $(n=11)$ stenting (applied devices $=$ Maverick balloon, Wingspan stent, and Liberté stent (all from Boston Scientific Corp, Natick, MA, USA), (3) Goose neck snare
(Microvena Corp, White Bear Lake, MN, USA), (4) sole manual aspiration $(n=8)$, (5) extensive microwire manipulation $(n=6)$, (6) Merci retriever X6 (Concentric Medical Inc, Mountain View, CA, USA; $n=4)$, (7) Neuronet (Guidant Corp, Temecula, CA, USA; $n=5$ ), and (8) catch device (Balt Extrusion Inc, Montmorency, France; $n=1$ ). Thirteen patients were treated by more than one device. The greatest number of devices used in one patient was three. The final recanalizing device was AngioJet in 25 cases, PTA with stenting in three cases, PTA without stenting in seven cases, Goose neck snare in six cases, sole manual aspiration in six cases, extensive microwire manipulation in six cases, Merci retriever X6 in three cases, Neuronet in three cases, and catch device in one case. Additional intra-arterial recombinant tissue plasminogen activator (rt-PA) was applied in 16 of 60 patients. These patients were treated between 2001 and 2008. Group $2(n=21)$ comprised patients who were treated by stent retriever (Solitaire stent, $n=11$ ) or thrombus fragmentation and aspiration (Penumbra System, Penumbra Inc, Alameda, CA, USA; $n=10$ ) alone. Additional intra-arterial rt-PA (Actilyse, Boehringer Ingelheim Pharma Gmbh \& Co KG, Ingelheim am Rhein, Germany) was applied in 2 of 21 patients. Patients in group 2 underwent MT between 2008 and May 2011.

All patients underwent comprehensive clinical examination by an experienced stroke neurologist and showed symptoms that were consistent with a severe acute brainstem syndrome (decreased consciousness, oculomotor dysfunction, hemiparesis/tetraparesis, and/or ataxia). Clinical outcome was measured by the modified Rankin scale (mRS) at the time of discharge or transfer of the patient and was compared between both groups. Favourable outcome was defined as mRS scores 0 through 3 .

\section{Neuroradiologic Diagnostics}

After neurological examination, at least one non-contrast computed tomography (CT) scan was performed to exclude large ischemic infarction, intracranial bleeding, and significant mass effect. Mostly, additional CT-angiography was available to prove $\mathrm{BAO}$ and visualize collateral flow by way of the posterior communicating arteries as well as additional sites of arterial occlusions due to early thrombus migration. In eight cases, magnetic resonance imaging (MRI) and MR-angiography, including diffusion-weighted imaging, were performed initially. Within $24 \mathrm{~h}$ after the endovascular procedure, a noncontrast $\mathrm{CT}$ scan was performed.

The presence of subarachnoid hemorrhage (SAH) was documented. Regarding parenchymal hyperdensities on postprocedural CT scans, the differentiation between postangiographic contrast-agent pooling and hemorrhage was 
made by means of serial CT scanning or MRI. On CT scans, hyperdensities that remained visible $<24 \mathrm{~h}$ after angiography were considered to be hemorrhage. The etiology of BAO was based on the findings of the angiographic study and additional diagnostic cardiological workup (i.e., electrocardiography, echocardiography). We subdivided the type of occlusion into four categories: (1) atherothrombotic, (2) artery-to-artery embolism from the proximal vertebral artery (VA; V0-V2 segments), (3) embolism from the heart or aortic arch, and (4) dissection of the VA. Atherothrombotic category included occlusion of the basilar artery (BA) combined with hemodynamically significant stenosis ( $>50$ lumen-diameter decrease) of the intracranial vertebrobasilar circulation (i.e., distal VA [V4 segment], vertebrobasilar junction, or the midbasilar artery) due to atherosclerosis. Embolism from the proximal VA was assumed if stenosis $(>50$ lumen-diameter decrease) of the extracranial cervical segment of the VA was present and the contralateral VA was occluded or hypoplastic.

\section{Endovascular Procedure}

All patients were treated under general anaesthesia. After inserting a $5 \mathrm{~F}$ to $8 \mathrm{~F}$ sheath into the right femoral artery, diagnostic intra-arterial digital subtraction angiography was performed with a $5 \mathrm{~F}$ to $8 \mathrm{~F}$ coaxial catheter on a high-resolution biplane angiography system with a matrix of $1,024 \times 1,024$ pixels. Catheters with larger diameters were used to block the artery for flow control. Usually the coaxial catheter was placed with the tip in the distal V2 segment approximately $1 \mathrm{~cm}$ below the atlas loops of the dominant VA. BAO was confirmed by diagnostic angiographic series with at least two projections. The site of occlusion was classified according to Archer and Horenstein [16] after the three anatomic segments of the BA and intracranial VA. In some cases, an additional flexible $4 \mathrm{~F}$ to $5 \mathrm{~F}$ intracranial catheter was used for distal access, distal thromboaspiration, and increased stability during clot removal. Mechanical recanalization was performed by using the devices mentioned previously. Proximal thromboaspiration creating a backflow in the BA was performed in all cases in group 2 using the automatic pump included in the Penumbra system in all cases treated with the Penumbra Separator and using manual aspiration in all cases treated with the Solitaire stent retriever. Before flexible aspiration catheters to enter the BA became available, in several cases of group 1, both vertebral arteries were catheterised and simultaneous bilateral aspiration was applied. It was used as bilateral proximal aspiration alone or in combination with retrievers. Overall, manual aspiration was performed in 31 patients in group 1 (16 bilaterally, 15 unilaterally). Additional local fibrinolysis was administered if necessary.
The procedure was stopped when the V4 segments, the entire BA, and the precommunicating segments of the posterior cerebral arteries (PCAs) were recanalized. This was defined as "complete recanalization." "Partial recanalization" was defined as remaining nonocclusive thrombotic material in the BA and/or persisting occlusion of the V4 segments and/or the precommunicating segments of the PCAs. "Needle-to-recanalization time" was determined as the interval from groin puncture to successful recanalization. All endovascular procedures were performed by trained interventional neuroradiologists from the Department of Neuroradiology. The treatment was performed under systemic heparin administration (activated clotting time $200-250 \mathrm{~s}$ ). All procedures were performed in accordance with our institutional guidelines. All neuroradiological examinations (CT, MRI, diagnostic angiography, and endovascular procedure) were re-evaluated in consensus by two experienced neuroradiologists who were unaware of the clinical findings.

For statistical analysis, the software package SPSS 19.0 was used. We applied nonparametric Mann-Whitney $U$ test for comparison of continuous variables and two-sided Fisher's exact test for comparison of proportions.

\section{Results}

The baseline characteristics of the patients are listed in Table 1. In group 2, cardioembolism was the most frequent etiology of stroke, whereas atherothrombotic origin of BAO was most often found in group 1. Before MT, fibrinolytic or antithrombotic drugs were more often administered in group 2 than in group 1 ("bridging therapy") (not significant, $p=0.614)$. More than half of patients in group 2 received intravenous (IV) rt-PA before MT $(p=0.03)$.

\section{Recanalization}

High recanalization rates of $95 \%$ were found in both groups. Complete recanalization was achieved slightly more often in group 1 than in group 2 (86.7 vs. 81, not significant, $p=0.498$ ). Needle-to-recanalization time was less than half in group 2 compared with group 1 (132.3 vs. $54.6 \min , p=0.01$ ). Details are listed in Table 2 .

Procedure-Related Complications and Distal Embolization

Procedure-related dissection $(n=5)$ and SAH $(n=9)$ occurred in group 1 but not in group $2(p=0.016)$ (Table 2). Three dissections were located in the BA and caused flow-limiting stenosis. Two minor dissections without relevant stenosis occurred in the V3 segment of the 
Table 1 Baseline characteristics a Two-sided Mann-Whitney

$U$ test

b Two-sided Fisher's exact test

\begin{tabular}{|c|c|c|c|}
\hline Baseline characteristics & Group $1(N=60)$ & Group $2(N=21)$ & $p$ \\
\hline \multicolumn{4}{|l|}{ Age (years) } \\
\hline Mean (SD) & $60.7(14.9)$ & $64.0(15.1)$ & \multirow[t]{2}{*}{$0.425^{\mathrm{a}}$} \\
\hline Median (range) & $64(17-89)$ & $70(35-90)$ & \\
\hline Women (\%) & 31.7 & 47.6 & $0.200^{\mathrm{b}}$ \\
\hline \multicolumn{4}{|l|}{ Etiology } \\
\hline Cardioembolism & $19(31.7 \%)$ & $15(71.4 \%)$ & $0.002^{\mathrm{b}}$ \\
\hline Embolism from VA & $11(18.3 \%)$ & $4(19.1 \%)$ & $1.000^{\mathrm{b}}$ \\
\hline Atherothrombotic & $29(48.3 \%)$ & $2(9.5 \%)$ & $0.002^{\mathrm{b}}$ \\
\hline Dissection & $1(1.7 \%)$ & $0(0 \%)$ & $1.000^{\mathrm{b}}$ \\
\hline \multicolumn{4}{|l|}{ Location of occlusion } \\
\hline Distal $1 / 3$ and/or top of BA & $22(36.7 \%)$ & $8(38.1 \%)$ & $1.000^{\mathrm{b}}$ \\
\hline Midbasilar & $9(15 \%)$ & $10(47.6 \%)$ & $0.005^{\mathrm{b}}$ \\
\hline Caudal & $29(48.3 \%)$ & $3(14.3 \%)$ & $0.009^{\mathrm{b}}$ \\
\hline \multicolumn{4}{|l|}{ Bridging } \\
\hline No bridging & $31(52.7 \%)$ & $9(43.6 \%)$ & $0.614^{\mathrm{a}}$ \\
\hline rt-PA & $10(16.7 \%)$ & $11(52.4 \%)$ & $0.03^{\mathrm{a}}$ \\
\hline GP IIb/IIIa inhibitor & $11(18.3 \%)$ & $0(0 \%)$ & $0.058^{\mathrm{a}}$ \\
\hline IV heparin & $11(18.3 \%)$ & $1(4.8 \%)$ & $0.170^{\mathrm{a}}$ \\
\hline MT devices used & $\begin{array}{l}\text { AngioJet, PTA, } \\
\text { Catch, Goose neck } \\
\text { snare, Neuronet, } \\
\text { Merci X6, sole } \\
\text { aspiration, } \\
\text { extensive wire } \\
\text { manipulation }\end{array}$ & Solitaire, Penumbra system & \\
\hline
\end{tabular}

Table 2 Recanalization results and procedure-related complications

\begin{tabular}{|c|c|c|c|}
\hline Results and complications & $\begin{array}{l}\text { Group 1 } \\
(N=60)\end{array}$ & $\begin{array}{l}\text { Group 2 } \\
(N=21)\end{array}$ & $p$ \\
\hline Recanalization (overall) & $57(95 \%)$ & $20(95.3 \%)$ & $1.000^{\mathrm{a}}$ \\
\hline Complete & $52(86.7 \%)$ & $17(81 \%)$ & $0.498^{\mathrm{a}}$ \\
\hline Partial & $5(8.3 \%)$ & $3(14.3 \%)$ & $0.421^{\mathrm{a}}$ \\
\hline $\begin{array}{l}\text { Mean }(\mathrm{SD}) \text { time to } \\
\text { recanalization }(\mathrm{min})\end{array}$ & $132.3(67.7)$ & $54.6(40.5)$ & $0.01^{\mathrm{a}}$ \\
\hline Dissection & $5(8.3 \%)$ & $0(0 \%)$ & $0.320^{\mathrm{a}}$ \\
\hline Subarachnoid hemorrhage & $9(15 \%)$ & $0(0 \%)$ & $0.103^{\mathrm{a}}$ \\
\hline $\begin{array}{l}\text { All procedure-related } \\
\text { complications } \\
\text { (dissection and SAH) }\end{array}$ & $14(23.3 \%)$ & $0(0 \%)$ & $0.016^{\mathrm{a}}$ \\
\hline Distal embolisation & $28(46.7 \%)$ & $10(47.6 \%)$ & $1.000^{\mathrm{a}}$ \\
\hline
\end{tabular}

a Two-sided Fisher's exact test

VA. Of the cases with dissections, favourable outcome was achieved in three patients (all $\mathrm{mRS}=3$ ), whereas one patient died. In 7 of 9 patients with intervention-associated subarachnoid hemorrhage, Angiojet was the recanalizing device; bridging therapy was applied in 5 of these 9 patients using IV rt-PA $(n=2)$, GP IIb/IIIa inhibitor $(n=2)$, and heparin $(n=1)$. In the remaining two cases,
SAH occurred after application of Merci retriever X6 and PTA, respectively. Six of the patients with SAH died, and only one patient had favourable outcome $(\mathrm{mRS}=3)$.

Frequency of distal embolization was nearly equivalent in both groups. No technical failure (e.g., device fracture) was observed.

\section{Clinical Outcome}

Clinical outcome as measured by mRS did not differ significantly between both groups. Favourable outcome was achieved in $33 \%$ of the cases in group 1 and in $28 \%$ of the cases in group $2(p=0.777)$. Mortality was $37 \%$ in group 1 and $33 \%$ in group $2(p=1.000)$.

\section{Discussion}

Several MT devices for recanalization of large intracranial artery occlusion have been introduced during the last 10 years. To our best knowledge, systematic data on safety of MT devices comparing different generations of recanalization devices and techniques are not available yet. In our study, reflecting "real-world" experience, we could show that modern stent retriever and suction thrombectomy 
provide a more rapid and safer removal of thromboembolic material in BAO compared with previous MT devices from 2001 through 2008.

In previous studies on intra-arterial fibrinolysis, recanalization rates of approximately $50-60 \%$ for complete recanalization, and $\leq 70 \%$ when combining partial and complete recanalization, were reported in acute BAO [16]. Until now, only anecdotal reports and small singlecenter studies on MT in BAO have reported promising recanalization rates of $72-100 \%[7,11,17,18]$. In the present study, partial or complete recanalization was achieved in $95 \%$ of cases in both groups.

One of the major advantages of MT is the rapidity with which the BA is reopened. In accordance with the literature [19] as well as our own experience, the time required for sole chemical thrombolysis is usually between 1 and $2.5 \mathrm{~h}$ in addition to the time for diagnostic angiography and positioning of the microcatheter. Similar to intra-arterial fibrinolysis, we found a needle-to-recanalization time of $132 \mathrm{~min}$ for MT devices used during the years 2001-2008. Modern MT systems showed a significant decrease in this time to a mean of $54 \mathrm{~min}$. Mean procedure times as low as 37-48 $\mathrm{min}$ were found in patients with large artery occlusion of the anterior and posterior circulation treated with the Solitaire stent $[15,20,21]$. Although we did not analyze the number of retrieval attempts in detail, the significant difference in recanalization time between both groups is likely to be caused by the low number of passes with stent retriever or suction thrombectomy compared with previous devices as well as by the simple handling of the new devices. Vessel rupture by the microwire and remote vessel traction injury causing subarachnoid bleeding as well as local vessel dissection are well known potentially hazardous procedure-related complications [12, 22]. In recent single center-studies [15, 21, 23, 24] on the safety and efficacy of the Solitaire stent, procedure-related complications were observed with a frequency of only $0-12 \%$. This is in accordance with our results because we found no vessel dissection and/or SAH in patients treated with Solitaire stent retriever or suction thrombectomy but in $14(23 \%)$ of 60 patients treated with previously used MT devices $(p=0.016)$. This significant difference is most likely the result of the easier application of the new MT devices and faster recanalization.

Loh et al. [25] analysed the number of retrieval attempts with the Merci retriever, after which the probability of iatrogenic complications increased in 94 patients with large cerebral artery occlusions. The investigators found that the rate of procedural complications increased from 14.8 to $42.4 \%$ when the number of three attempts was exceeded regardless of final recanalization outcome [25]. This might explain the significantly greater incidence of procedurerelated complications in group 1 in our study according to neurointerventionalists' rule, i.e., "the longer the procedure, the more frequent the complications."

Unlike local thrombolysis, MT is considered to prevent distal embolization of thrombus fragments. According to the data of Schulte-Altedorneburg et al. [5], occlusion of the postcommunicating segment of one or both PCAs due to distal embolization occurs in $39 \%$ of patients with successful intra-arterial fibrinolysis of the BA. In an in vitro study, Liebig et al. [26] compared five embolectomy systems [Catch, Merci, InTime, and Attracter (Boston Scientific Corp, Natick, MA, USA) and Phenox clot retriever (Phenox GmbH, Bochum, Germany)] and found frequent thrombus fragmentation as well as thrombus migration in all devices. In another experimental study, it has been shown that mechanical embolectomy may cause even more embolic debris than chemical thrombolysis if used without flow reversal [27]. Although we regularly applied remote or local thromboaspiration during mechanical retraction maneuvers, distal thrombus migration was frequent in both groups (46.7 vs. $47.6 \%$ ). However, considering the severity and morbidity of BAO, partial thrombus migration into one or two cerebellar arteries or distal segments of the PCA is usually not decisive for patients' prognosis if the target vessels, BA, and precommunicating arteries are reopened. Furthermore, additional selective low-dose intra-arterial administration of rt-PA into cerebellar arteries or PCAs is still a sufficient option in these cases.

Bridging therapy, i.e., the combination of intravenous thrombolysis with rt-PA, GP IIb/IIIa inhibitors, or, previously, heparin after endovascular reperfusion therapy has been reported in BAO before $[17,18,28]$. It combines the early onset of IV thrombolysis with the high recanalization rates of MT. In a previous study, we retrospectively evaluated the outcome of BAO patients referred from community hospitals [17]. One group received bridging therapy consisting of IV rt-PA during transfer and subsequent MT at the neurovascular center. The patients of the other group were transferred without IV recanalizing therapy. The functional outcome of patients treated by bridging therapy was significantly better than that of patients treated with MT alone. In the present study, we did not compare the clinical outcome of both groups, but one might argue that the greater rate of bridging therapy with IV rt-PA in group 2 is another reason for the safer and faster recanalization in these patients [29]. Therefore, the results encourage us to persist with the cooperative treatment protocol "drip, ship, and retrieve" implemented by Pfefferkorn et al. [17].

The study has limitations, partly due to its retrospective design. First, the recanalization results of both groups were influenced by learning curves regarding the application of complex MT systems, especially in group 1. Second, the choice and the order of the MT devices, if more than one 
were used, were heterogeneous and not standardised in group 1. Third, the intravenous substances administered for bridging therapy were more inhomogeneous in group 1 than in group 2.

We did not strengthen clinical aspects for several reasons. First, our purpose was to focus on the evolvement of technical efficacy and technical proceedings of mechanical recanalisation in BAO during the last 10 years. Second, unlike anterior circulation ischemia, exact onset of symptoms was mostly difficult to determine due to the oftenfluctuating symptoms or prodromi in vertebrobasilar ischemia. Several patients were transferred to our clinic from community hospitals in a comatose state. Thus, determination of time window and preinterventional National Institute of Health Stroke Scale was inhomogeneous and difficult to exactly verify. Despite the decrease of intervention-associated complications by the new devices, favourable clinical outcomes did not differ in our study. In our opinion, this is a limitation of our study caused by the retrospective design.

\section{Conclusion}

We could show that MT has become a safe, rapid, and effective procedure with high recanalization rates and a low risk of procedure-related and potentially harmful complications in BAO during the last decade. Modern stent retriever and thrombus separation and aspiration seem to have enabled interventional neuroradiologists to overcome the previous limitations of hemorrhagic complications and iatrogenic vessel wall injuries.

Conflict of interest Markus Holtmannspoetter reports personal fees for proctor and consultant services from Covidien, personal fees for proctor and consultant services from Sequent Medical, and personal fees for consultant services from Microvention outside of the submitted work. Gunther Fesl, Maximilian Patzig, Thomas E. Mayer, Thomas Pfefferkorn, Hartmut Brückmann, Gernot Schulte-Altedormeburg have no conflict of interest.

\section{References}

1. Zeumer H, Hacke W, Ringelstein EB (1983) Local intraarterial thrombolysis in vertebrobasilar thromboembolic disease. AJNR Am J Neuroradiol 4(3):401-404

2. Hacke W, Zeumer H, Ferbert A, Brückmann H, Del Zoppo GJ (1988) Intra-arterial thrombolytic therapy improves outcome in patients with acute vertebrobasilar occlusive disease. Stroke 19(10):1216-1222

3. Schulte-Altedorneburg G, Hamann GF, Mull M et al (2006) Outcome of acute vertebrobasilar occlusions treated with intraarterial fibrinolysis in 180 patients. AJNR Am J Neuroradiol 27(10):2042-2047
4. Eckert B, Kucinski T, Pfeiffer G, Groden C, Zeumer H (2003) Endovascular therapy of acute vertebrobasilar occlusion: early treatment onset as the most important factor. Cerebrovasc Dis 15(4):258-263

5. Schulte-Altedorneburg G, Brückmann H, Hamann GF et al (2007) Ischemic and hemorrhagic complications following intraarterial fibrinolysis in vertebrobasilar occlusion. AJNR Am J Neuroradiol 28(2):378-381

6. Cross DT III, Derdeyn CP, Moran CJ (2001) Bleeding complications after basilar artery fibrinolysis with tissue plasminogen activator. AJNR Am J Neuroradiol 22(3):521-525

7. Mayer TE, Hamann GF, Schulte-Altedorneburg G, Brückmann H (2005) Treatment of vertebrobasilar occlusion by a coronary waterjet thrombectomy device-a pilot study. AJNR Am J Neuroradiol 26(6):1389-1394

8. Penumbra Pivotal Stroke Trial Investigators (2009) The Penumbra Pivotal Stroke Trial: safety and effectiveness of a new generation of mechanical devices for clot removal in intracranial large vessel occlusive disease. Stroke 40(8):2761-2768

9. Smith WS, Sung G, Saver J, The Multi MERCI Investigators et al (2008) Mechanical thrombectomy for acute ischemic stroke: final results of the Multi MERCI trial. Stroke 39(4):1205-1212

10. Fields JD, Lutsep HL, Smith WS, MERCI Multi MERCI Investigators (2011) Higher degrees of recanalization after thrombectomy for acute stroke are associated with improved outcome and decreased mortality: pooled analysis of the MERCI and multi MERCI trials. AJNR Am J Neuroradiol 32(11):2170-2174

11. Chapot R, Houdart E, Rogopoulos A, Mounayer C, Saint-Maurice JP, Merland JJ (2002) Thromboaspiration in the basilar artery: report of two cases. AJNR Am J Neuroradiol 23(2):282-284

12. Yin NS, Benavides S, Starkman S et al (2010) Autopsy findings after intracranial thrombectomy for acute ischemic stroke. A clinicopathologic study of 5 patients. Stroke 41(5):938-947

13. Castano C, Serena J, Davalos A (2009) Use of the new Solitaire $\mathrm{AB}$ device for mechanical thrombectomy when the MERCI clot retriever has failed to remove the clot: a case report. Interv Neuroradiol 15(2):209-214

14. Castano C, Dorado L, Guerrero C et al (2010) Mechanical thrombectomy with the Solitaire $\mathrm{AB}$ device in large artery occlusions of the anterior circulation. A pilot study. Stroke 41(8): 1836-1840

15. Stampfl S, Hartmann M, Ringleb PA, Haehnel S, Bendszus M, Rohde S (2011) Stent placement for flow restoration in acute ischemic stroke: a single-center experience with the Solitaire stent system. AJNR Am J Neuroradiol 32(7):1245-1248

16. Archer CR, Horenstein S (1977) Basilar artery occlusion. Clinical and radiological correlation. Stroke 8(3):383-390

17. Pfefferkorn T, Holtmannspötter M, Schmidt C et al (2010) Drip ship and retrieve. Cooperative recanalization therapy in acute basilar artery occlusion. Stroke 41(4):722-726

18. Eckert B, Koch C, Thomalla G et al (2005) Aggressive therapy with intravenous abciximab and intra-arterial rtPA and additional PTA/stenting improves clinical outcome in acute vertebrobasilar occlusion. Combined local fibrinolysis and intravenous abciximab in acute vertebrobasilar stroke treatment (FAST) - results of a multicenter study. Stroke 36(6):1160-1165

19. Furlan A, Higashida R, Wechsler L et al (1999) Intra-arterial prourokinase for acute ischemic stroke. The PROACT II study: a randomized controlled trial. Prolyse in acute cerebral thromboembolism. JAMA 282(21):2003-2011

20. Park H, Hwang G, Jin SC et al (2011) A retrieval thrombectomy technique with the Solitaire stent in a large cerebral artery occlusion. Acta Neurochir 153(8):1625-1631

21. Machi P, Costalat V, Lobotesis K et al (2012) Solitaire FR thrombectomy system: immediate results in 56 consecutive ischemic stroke patients. J Neurointerv Surg 4(1):62-66 
22. Jahan R (2010) Solitaire flow-restoration device for treatment of acute ischemic stroke: safety and recanalization efficacy study in a swine vessel occlusion model. AJNR Am J Neuroradiol 31(10):1938-1943

23. Miteff F, Faulder KC, Goh ACC, Steinfort BS, Sue C, Harrington TJ (2011) Mechanical thrombectomy with a self-expanding retrievable intracranial stent (Solitaire $\mathrm{AB}$ ): experience in 26 patients with acute cerebral artery occlusion. AJNR Am J Neuroradiol 32(6): 1078-1081

24. Fesl G, Patzig M, Holtmannspoetter M et al (2011) Endovascular mechanical recanalisation of acute carotid-T occlusions: a singlecenter retrospective analysis. Cardiovasc Intervent Radiol 34(2): 280-286

25. Loh Y, Jahan R, McArthur DL et al (2010) Recanalization rates decrease with increasing thrombectomy attempts. AJNR Am J Neuroradiol 31(5):935-939

26. Liebig T, Reinartz J, Hannes R, Miloslavski M, Henkes H (2008) Comparative in vitro study of five mechanical embolectomy systems: effectiveness of clot removal and risk of distal embolization. Neuroradiology 50(1):43-52

27. Greenberg RK, Ouriel K, Srivastava S et al (2000) Mechanical versus chemical thrombolysis: an in vitro differentiation of thrombolytic mechanisms. J Vasc Interv Radiol 11(2 Pt 1): 199-205

28. Rubiera M, Ribo M, Pagola J et al (2011) Bridging intravenousintra-arterial rescue strategy increases recanalization and the likelihood of a good outcome in nonresponder intravenous tissue plasminogen activator-treated patients. A case-control study. Stroke 42(4):993-997

29. Pfefferkorn T, Holtmannspötter M, Patzig M et al (2012) Preceding intravenous thrombolysis facilitates endovascular mechanical recanalization in large intracranial artery occlusion. Int J Stroke 7(1):14-18 